\title{
Flexor pollicis longus tendon rupture by sandwiched underlying volar locking plate and distal radius
}

Takuya Uemura, Tadashi Okano, Ema Onode, Takuya Yokoi, Kosuke Shintani, Mitsuhiro Okada, Hiroaki Nakamura

\begin{tabular}{|c|l|}
\hline Citation & Journal of Medical Ultrasonics, 45(4);647-651 \\
\hline Issue Date & $2018-10$ \\
\hline Type & Journal Article \\
\hline Textversion & Author \\
\hline Relation & $\begin{array}{l}\text { This is a post-peer-review, pre-copyedit version of an article published in Journal of } \\
\text { Medical Ultrasonics. The final authenticated version is available online at: } \\
\text { https://doi.org/10.1007/s10396-018-0882-1. }\end{array}$ \\
\hline DOI & \begin{tabular}{l}
$10.1007 / \mathrm{s} 10396-018-0882-1$ \\
\hline
\end{tabular} \\
\hline
\end{tabular}

Self-Archiving by Author(s)

Placed on: Osaka City University

UEMURA T., OKANO T., ONODE E., YOKOI T., SHINTANI K., OKADA M., \& NAKAMURA H. (2018). Flexor pollicis longus tendon rupture by sandwiched underlying volar locking plate and distal radius. Journal of Medical Ultrasonics. 45, 647-651. doi: 10.1007/s10396-018-0882-1 
Flexor pollicis longus tendon rupture by sandwiched underlying volar locking plate and distal radius: A case report

Takuya Uemura, MD, $\mathrm{PhD}^{1 *}$, Tadashi Okano, $\mathrm{MD}, \mathrm{PhD}^{1}$, Ema Onode, $\mathrm{MD}^{1}$, Takuya Yokoi, $\mathrm{MD}^{1}$, Kosuke Shintani MD ${ }^{1}$, Mitsuhiro Okada, $\mathrm{MD}, \mathrm{PhD}^{1}$, Hiroaki Nakamura, $\mathrm{MD}, \mathrm{PhD}^{1}$.

${ }^{1}$ Department of Orthopaedic Surgery, Osaka City University Graduate School of Medicine, 1-4-3 Asahimachi Abeno-ku, Osaka 545-8585, Japan.

*Corresponding author: Takuya Uemura, MD, PhD, Department of Orthopaedic Surgery, Osaka City University Graduate School of Medicine, 1-4-3 Asahimachi Abeno-ku, Osaka 545-8585, Japan. Tel: +81-6-6645-3851. Fax: +81-6-6646-6260. E-mail: t-uemura@med.osaka-cu.ac.jp 


\begin{abstract}
Flexor pollicis longus (FPL) tendon rupture is a major complication of volar locking plate fixation for distal radius fractures. The tendon rupture is usually caused by friction between the distal edge of the plate and the FPL tendon, and has been well detected recently with ultrasonography. However, rarely, the volar locking plate itself entraps the FPL tendon, leading to its rupture. A 63-year-old man was consistently unable to flex his right thumb after previous surgery for a distal radius fracture at another hospital. Ultrasonography demonstrated loss of tendon gliding and unusual patterns of the FPL tendon. The tendon was sandwiched between the plate and the distal radius, and was penetrated by the distal locking screw, which was comparable to intraoperative findings of complete entrapment and rupture of the FPL tendon from the underlying the plate. The tendon defects were repaired using a palmaris longus tendon graft after removing the screws and plate. Finally, he could flex his thumb actively with satisfaction. Unusual patterns of FPL tendon rupture buried under inadequate plate positioning must be recognized, as in this case. Ultrasonographic assessment is routinely recommended to visibly determine any FPL tendon damages after volar locking plate fixation for distal radius fractures.
\end{abstract}

Keywords: Flexor pollicis longus tendon, locking plate, ultrasonography, tendon rupture and distal radius fracture. 


\section{Introduction}

Distal radius fractures remain one of the most common orthopedic injuries, accounting for $8-17 \%$ of all extremity fractures, and open reduction and internal fixation with a volar locking plate has become the gold standard treatment to correct them [1-4]. However, several complications associated with volar locking plates have also become better defined $[2,5,6]$. Flexor pollicis longus (FPL) tendon rupture is one of the major complications, having an incidence rate of $0.2-12 \%$ [2, 5-7]. Most flexor tendon ruptures are caused by friction between the prominent volar distal edge of the plate and the flexor tendon. Recently, ultrasonography has become a useful method for early detection of FPL tendon attrition and prevention of FPL tendon rupture following volar locking plate fixation of distal radius fractures [8-12]. However, rarely, the volar locking plate itself entraps the FPL tendon entirely and causes its rupture, which has never been previously evaluated with ultrasonography. Here, we report a case of complete rupture of the FPL tendon, which was sandwiched between the underlying volar locking plate and the distal radius, treated with successful assessment of preoperative ultrasonography.

\section{Case report}

A 63-year-old man fell from a stepladder and sustained a right distal radius fracture. He underwent volar locking plate fixation surgery using Variable Angle-LCP Two-Column Distal Radius Plates 2.4, narrow (Depuy Synthes Japan, Japan) through a trans-flexor carpi radialis approach without minimally invasive surgery at another hospital. As the wrist pain and swelling, like complex regional pain syndrome, remained after surgery, he was continuously treated with medication and rehabilitation therapy, although he had noticed difficulty in right thumb movement immediately after the operation. Four months after the operation, wrist pain and swelling improved, but he was referred to our hospital because he was consistently unable to flex the interphalangeal joint of his right thumb (Fig. 1). The bone healing of the distal radius fracture was achieved, based on radiographs, with lifting the distal edge of the plate from the volar aspect of the distal radius. Surgical treatment for the FPL tendon rupture was planned, with removal of the volar locking plate. Preoperative ultrasonography examination was performed using HI VISION Ascendus (Hitachi Medical Corporation, Japan) with a multifrequency linear transducer (18-6 MHz) to find the pathological damages of the FPL tendon, but showed that the FPL tendon was tightly entrapped between the plate and the distal radius (Fig. 2). Furthermore, during passive flexion of the thumb, slight movement of the slack FPL tendon was visibly only distal to the distal rim of the plate, in which distal locking screw were observed clearly, but obviously diminished proximally in real-time ultrasound imaging. Intraoperatively, the FPL tendon was found embedded entirely under the plate and the distal locking screw penetrated the tendon (Fig. 3). The proximal stump of the FPL tendon was shortened proximally through the window of the plate and was clearly observed on the plate. The screws and plate were removed, and the buried distal stump of the FPL tendon was released. After the debridement of the proximal stump of the FPL tendon, the $4 \mathrm{~cm}$ tendon defects were repaired with a free double-bundle tendon graft of the palmaris longus using interlacing sutures. Passive range of motion exercise of the thumb was initiated during occupational therapy immediately after the operation, and active range of motion exercise was started one week after the operation. After the 1-year follow-up, he was able to flex his thumb 
actively in the same manner as he could prior to the injury (Fig. 4).

\section{Discussion}

As volar locking plate fixation for distal radius fractures has increased in popularity, complications of this surgical treatment have also been increasing [2, 5, 6]. In particular, FPL tendon rupture is a critical complication, given its anatomic location, and leads to loss of hand function and requires additional surgical treatment $[11,13]$. To prevent FPL tendon rupture, intraoperative proper placement of the volar locking plate proximal to the watershed line of the distal radius with anatomical reduction is necessary [11, 14-16]. Repair of the pronator quadratus muscle with coverage of the plate is also important. Loss of reduction and inappropriate plate position, as in the present case, can cause FPL tendon attrition, resulting in its rupture. In the present case, the FPL tendon was probably sandwiched from the radial side during setting of the plate on the distal radius. Tendon retraction might have been insufficient, possibly because the operator did not have an assistant or failed to check the operative field while only watching the monitor on an X-ray image intensifier. Additionally, the distal locking screw was slightly longer and penetrated the dorsal cortex of the distal radius. In such cases, extensor tendon ruptures are more frequent than are flexor tendon ruptures. Therefore, it is important to evaluate damage not only of the flexor tendons but also of the extensor tendons.

In addition to intraoperative risk factors for the FPL tendon rupture mentioned above, other risk factors for FPL tendon rupture after volar locking plate fixation surgery have been identified. Soong et al. described that prominence of the plate over the watershed line in lateral radiographs was associated with an increased risk factor for FPL tendon rupture [17]. More recently, ultrasonographic assessment has identified visible FPL tendon attrition and damage following volar locking plate fixation. Nanno et al. revealed ultrasonographic FPL kinematics of FPL tendon movement during wrist and finger motions in patients with distal radius fractures who underwent volar locking plate procedures [12]. Kara et al. reported that increases in the thickness of the FPL tendon and a decrease in the distance between the tendon and the plate could be determined using ultrasonography after volar locking plate fixation, warning that asymptomatic tenosynovitis may eventually cause tendon ruptures [11]. Tanaka et al. emphasized the usefulness of color Doppler imaging to detect tendon attrition because it provided more visibility compared with other methods [8]. Tokunaga and Abe demonstrated asymptomatic erosion of the FPL tendon, which might be a predictor of tendon rupture, after volar locking plate fixation using ultrasonography, and recommended the use of ultrasonography to routinely evaluate FPL tendon damage [10]. Kadoma et al. also advocated that ultrasonography could be used to identify the type of intermediate tissue between the FPL tendon and the volar locking plate, suggesting that ultrasonography has the potential to predict the risk of attenuation of the FPL tendon [9]. On the other hand, Yamazaki et al. described that sonography might be difficult to employ as a diagnostic tool to judge the risk for flexor tendon attrition, but that a subdermal crepitus sound during thumb movement was an independent risk factor for flexor tendon attrition [18].

We also believe that ultrasonography is a useful method to assess FPL tendon damage after volar locking plate fixation for the distal radius. Although almost all previous cases showed friction between the FPL tendon and the plate on ultrasonographic images as mentioned above; the present case showed unusual ultrasonographic findings of an 
initially buried and ruptured FPL tendon underlying the plate. Entrapment of the FPL tendon by the volar locking plate itself is extremely rare, with only one case previously reported [19]. In this case, however, the plate partially compressed the FPL tendon after minimally invasive plate osteosynthesis and a partial tear in the FPL tendon was repaired using only direct sutures without preoperative ultrasonographic assessment. In contrast, we report the first case of complete entrapment and total rupture of the FPL tendon underlying a volar locking plate that was repaired with tendon grafting, after a definitive diagnosis using preoperative ultrasonography. Unusual patterns of FPL tendon rupture buried under inadequate plate positioning must be recognized, as in the present case. Ultrasonographic assessments should be routinely performed to visibly assess the FPL tendon damage after volar locking plate fixation for distal radius fractures.

\section{Acknowledgements: None.}

Ethical statements: All procedures followed were in accordance with the ethical standards of the responsible committee on human experimentation (institutional and national) and with the Helsinki Declaration of 1964 and later versions.

Informed consent: Informed consent was obtained from the patient in this study.

Conflicts of interest: Takuya Uemura, Tadashi Okano, Ema Onode, Takuya Yokoi, Kosuke Shintani, Mitsuhiro Okada, and Hiroaki Nakamura declare that they have no conflict of interest.

\section{References}

[1] Orbay JL, Touhami A. Current concepts in volar fixed-angle fixation of unstable distal radius fractures. Clin Orthop Relat Res. 2006;445:58-67.

[2] Monaco NA, Dwyer CL, Ferikes AJ, et al. Hand Surgeon Reporting of Tendon Rupture Following Distal Radius Volar Plating. Hand (N Y). 2016;11:278-86.

[3] Nana AD, Joshi A, Lichtman DM. Plating of the distal radius. J Am Acad Orthop Surg. 2005;13:159-71.

[4] Pogue DJ, Viegas SF, Patterson RM, et al. Effects of distal radius fracture malunion on wrist joint mechanics. J Hand Surg Am. 1990;15:721-7.

[5] Satake H, Hanaka N, Honma R, et al. Complications of Distal Radius Fractures Treated by Volar Locking Plate Fixation. Orthopedics. 2016;39:e893-6.

[6] Casaletto JA, Machin D, Leung R, et al. Flexor pollicis longus tendon ruptures after palmar plate fixation of fractures of the distal radius. J Hand Surg Eur Vol. 2009;34:471-4.

[7] Asadollahi S, Keith PP. Flexor tendon injuries following plate fixation of distal radius fractures: a systematic review of the literature. J Orthop Traumatol.

2013;14:227-34.

[8] Tanaka Y, Gotani H, Yano K, et al. Evaluation of flexor pollicis longus tendon attrition using color Doppler imaging after volar plate fixation for distal radius fracture. J Orthop Sci. 2017;22:447-52.

[9] Kadoma C, Takahara M, Maruyama M, et al. Ultrasonographic Assessment of the Flexor Pollicis Longus Tendon After Plate Fixation. Orthopedics. 2017;40:e104-e8. 
[10] Tokunaga S, Abe Y. Asymptomatic Flexor Tendon Damages after Volar Locking Plate Fixation of Distal Radius Fractures. J Hand Surg Asian Pac Vol. 2017;22:75-82. [11] Kara A, Celik H, Bankaoglu M, et al. Ultrasonic Evaluation of the Flexor Pollicis Longus Tendon Following Volar Plate Fixation for Distal Radius Fractures. J Hand Surg Am. 2016;41:374-80.

[12] Nanno M, Kodera N, Tomori Y, et al. Transverse ultrasound assessment of the flexor pollicis longus tendon movement on the distal radius during wrist and finger motion in distal radius fracture with volar plating. J Med Ultrason (2001). 2016;43:29-36.

[13] Limthongthang R, Bachoura A, Jacoby SM, et al. Distal radius volar locking plate design and associated vulnerability of the flexor pollicis longus. J Hand Surg Am. 2014;39:852-60.

[14] Orbay JL. The treatment of unstable distal radius fractures with volar fixation. Hand Surg. 2000;5:103-12.

[15] Adham MN, Porembski M, Adham C. Flexor tendon problems after volar plate fixation of distal radius fractures. Hand (N Y). 2009;4:406-9.

[16] Cross AW, Schmidt CC. Flexor tendon injuries following locked volar plating of distal radius fractures. J Hand Surg Am. 2008;33:164-7.

[17] Soong M, van Leerdam R, Guitton TG, et al. Fracture of the distal radius: risk factors for complications after locked volar plate fixation. J Hand Surg Am. 2011;36:3-9.

[18] Yamazaki H, Uchiyama S, Komatsu M, et al. Risk Assessment of Tendon Attrition Following Treatment of Distal Radius Fractures With Volar Locking Plates Using Audible Crepitus and Placement of the Plate: A Prospective Clinical Cohort Study. J Hand Surg Am. 2015;40:1571-81.

[19] Chiu YC, Kao FC, Tu YK. Flexor pollicis longus tendon entrapment after performing minimally invasive plate osteosynthesis of a distal radius fracture: a case report. Hand Surg. 2013;18:403-6. 


\section{Figure legends}

Figure 1.

The preoperative photograph depicts the inability of the patient to flex the interphalangeal joint of the right thumb (a). The lateral radiograph of the right wrist shows the lifting of the distal edge of the locking plate from the volar aspect of the distal radius $(b)$.

\section{Figure 1}

a

b

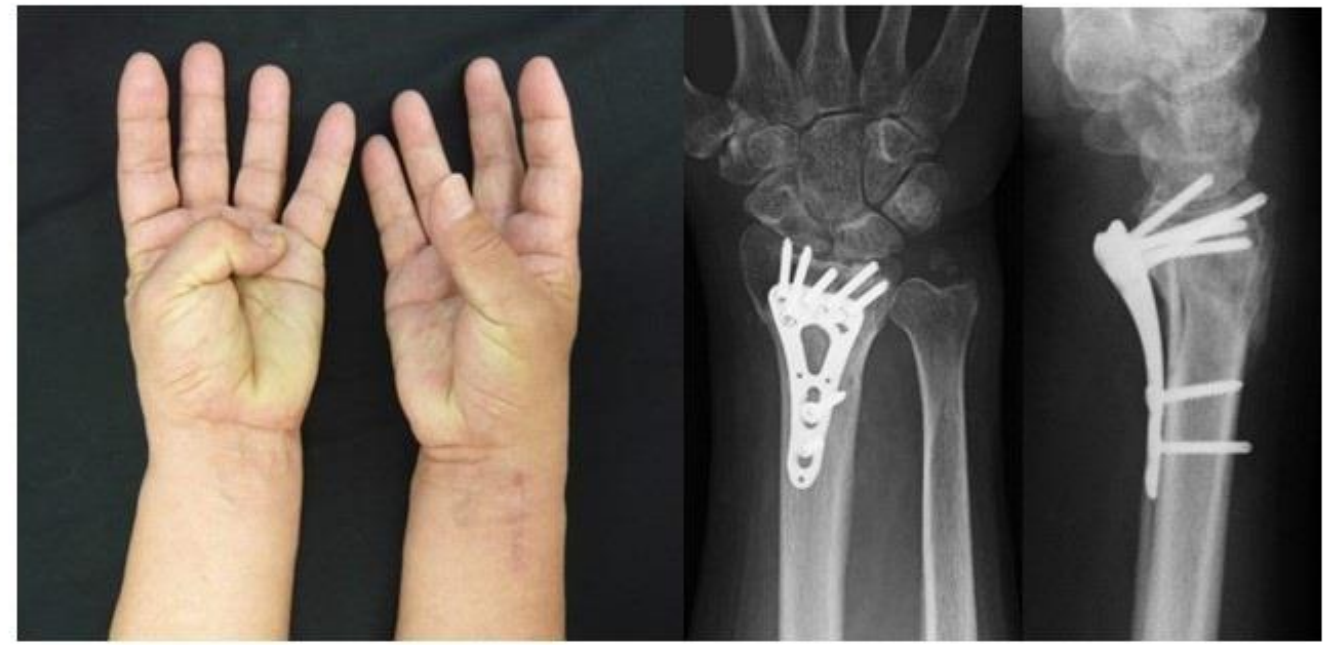


Figure 2.

The preoperative ultrasonography images (a) show that the proximal stump (arrowhead) of the flexor pollicis longus (FPL) tendon is interrupted by the plate and the distal stump (asterisk), which is sandwiched between the plate and the distal radius when compared with the computed tomography image (b). The movement of the slack FPL tendon during passive flexion of the thumb is diminished at the level in which the distal locking screw (arrow) is observed more clearly (c). 
Figure 2

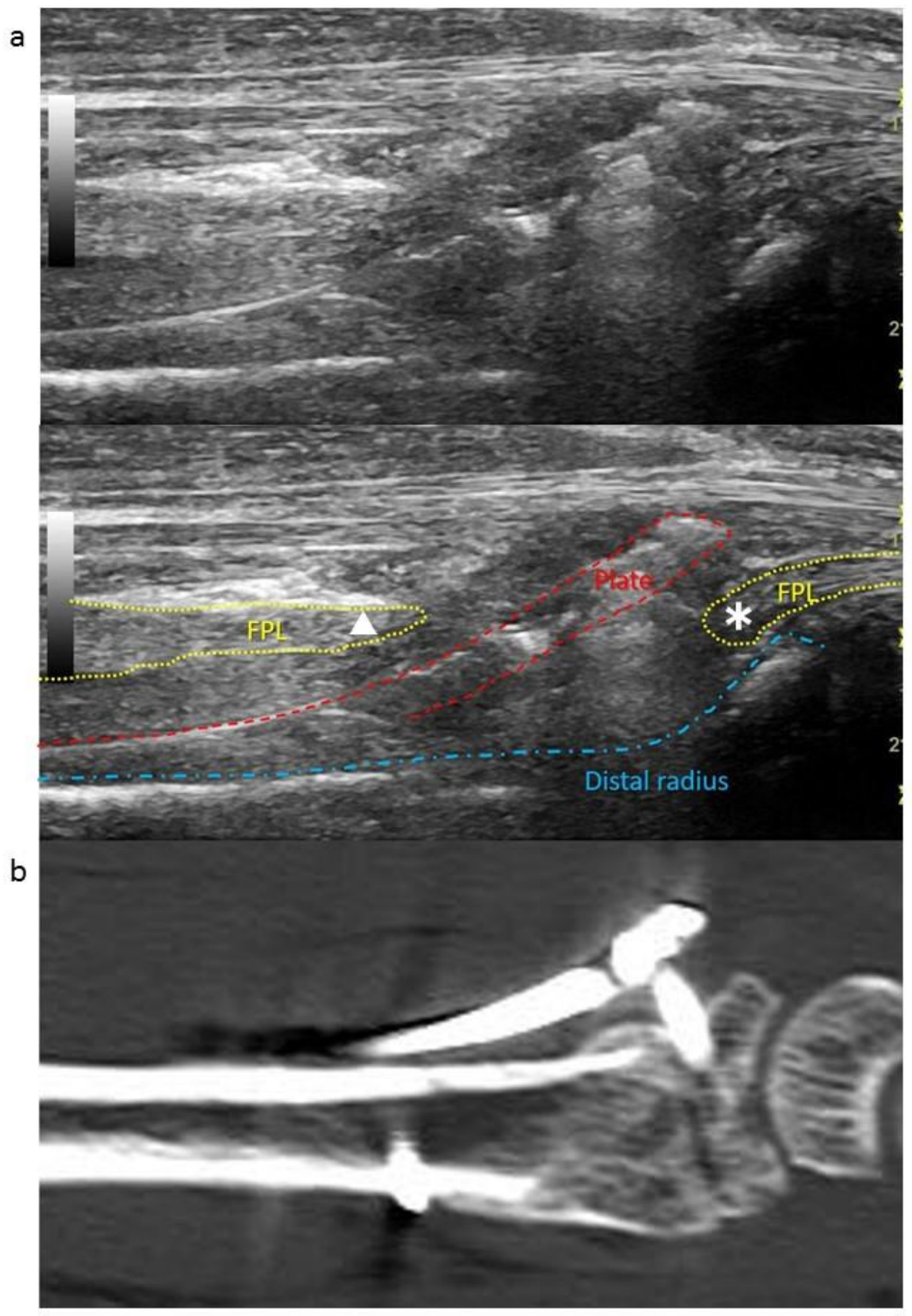




\section{Figure 2}

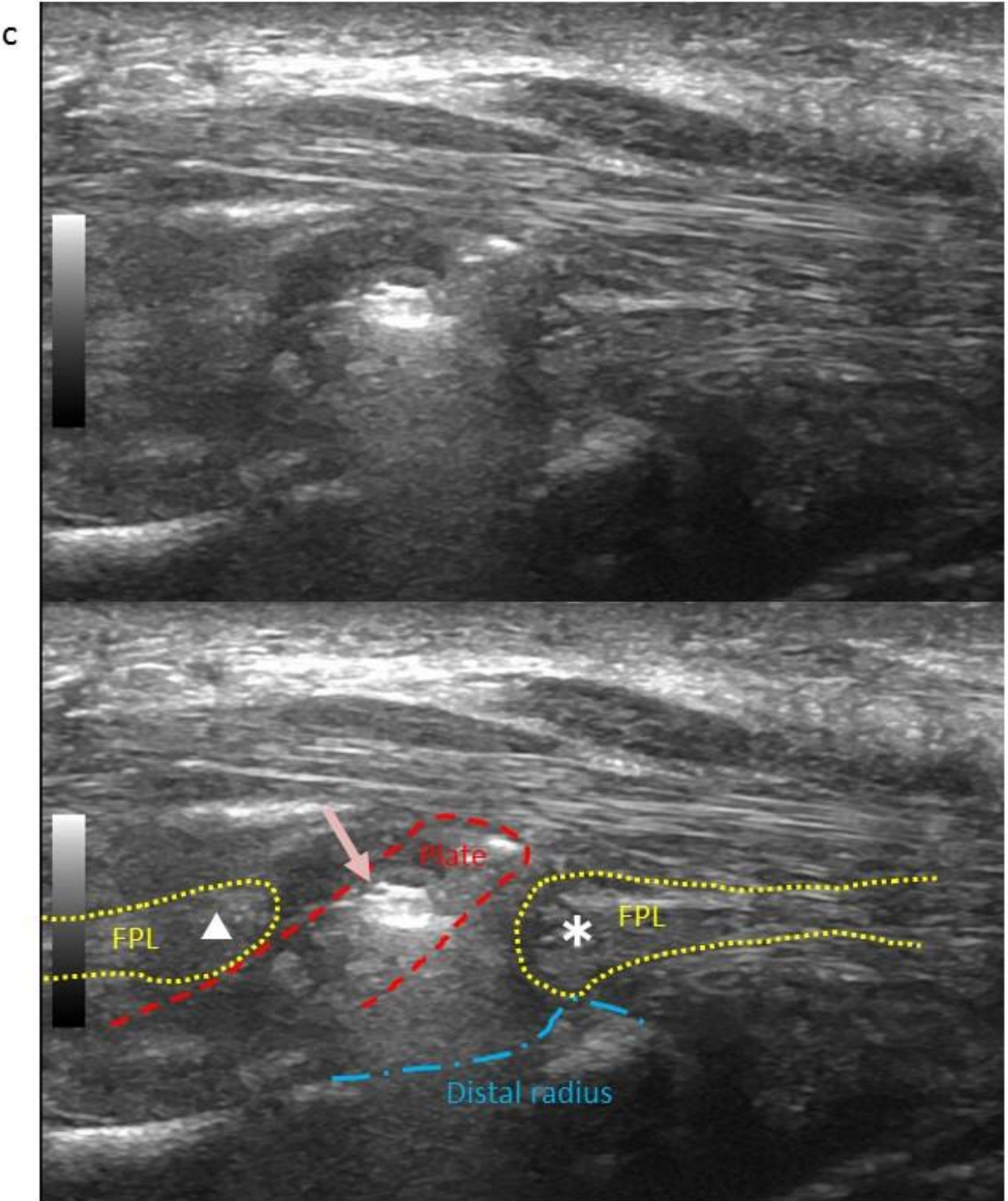


Figure 3.

Intraoperative findings show the flexor pollicis longus (FPL) tendon embedded under the plate (a), total tendon rupture of the FPL with the distal stump (asterisk) and proximal stump (arrowhead) after plate removal (b), and reconstructed tendon defects with palmaris longus tendon grafting (c). 
Figure 3

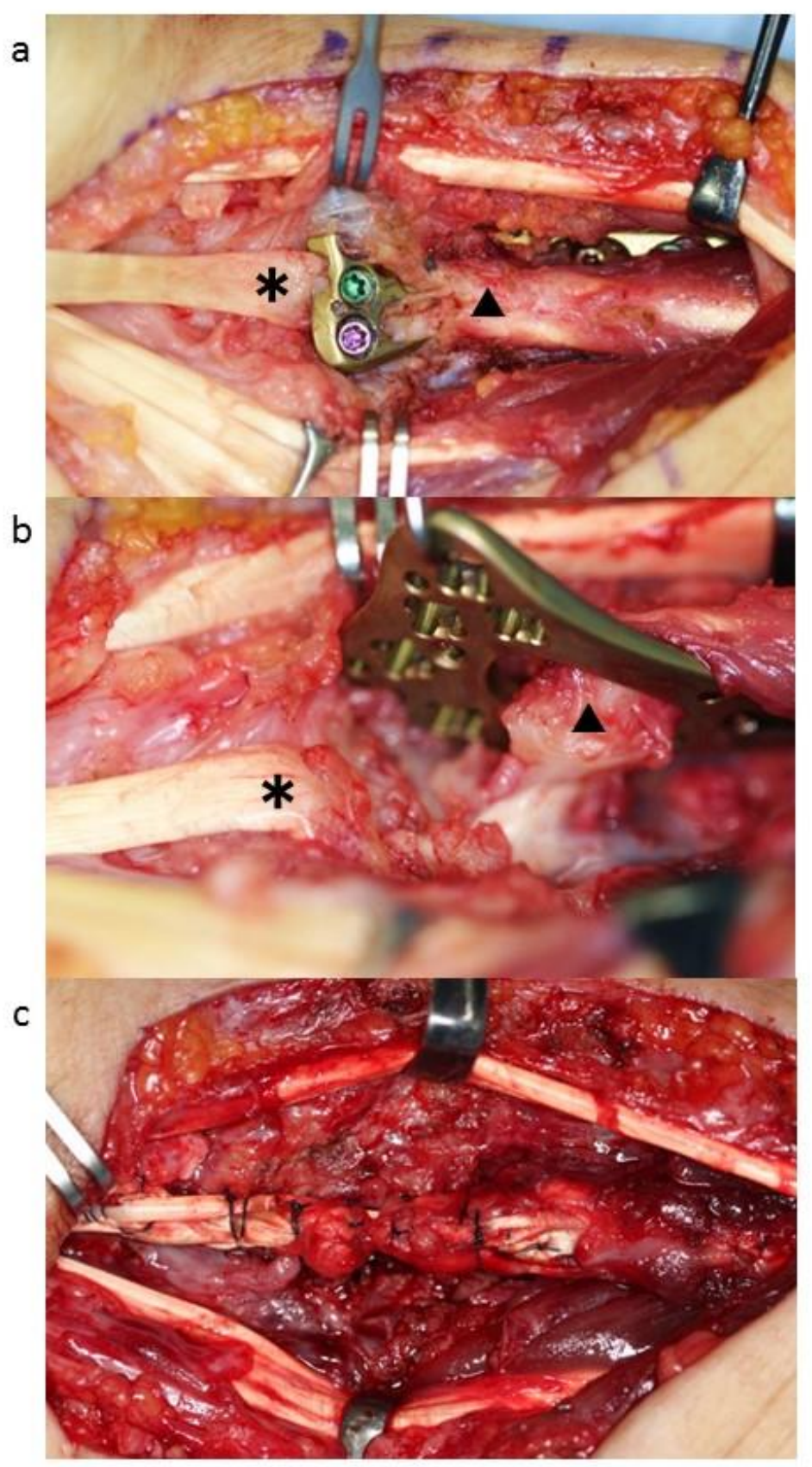


Figure 4.

Postoperative photographs depicting the ability of the patient to flex the interphalangeal joint of the right thumb (one year after the operation). 
Figure 4

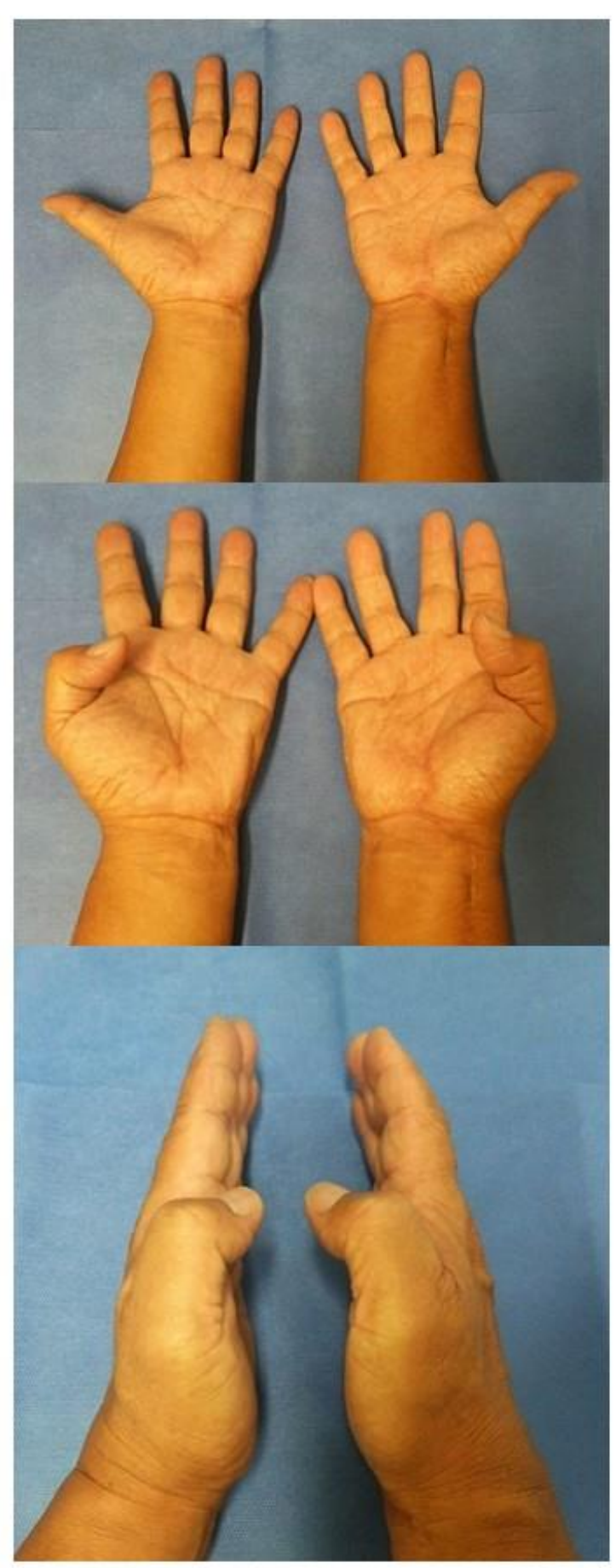

